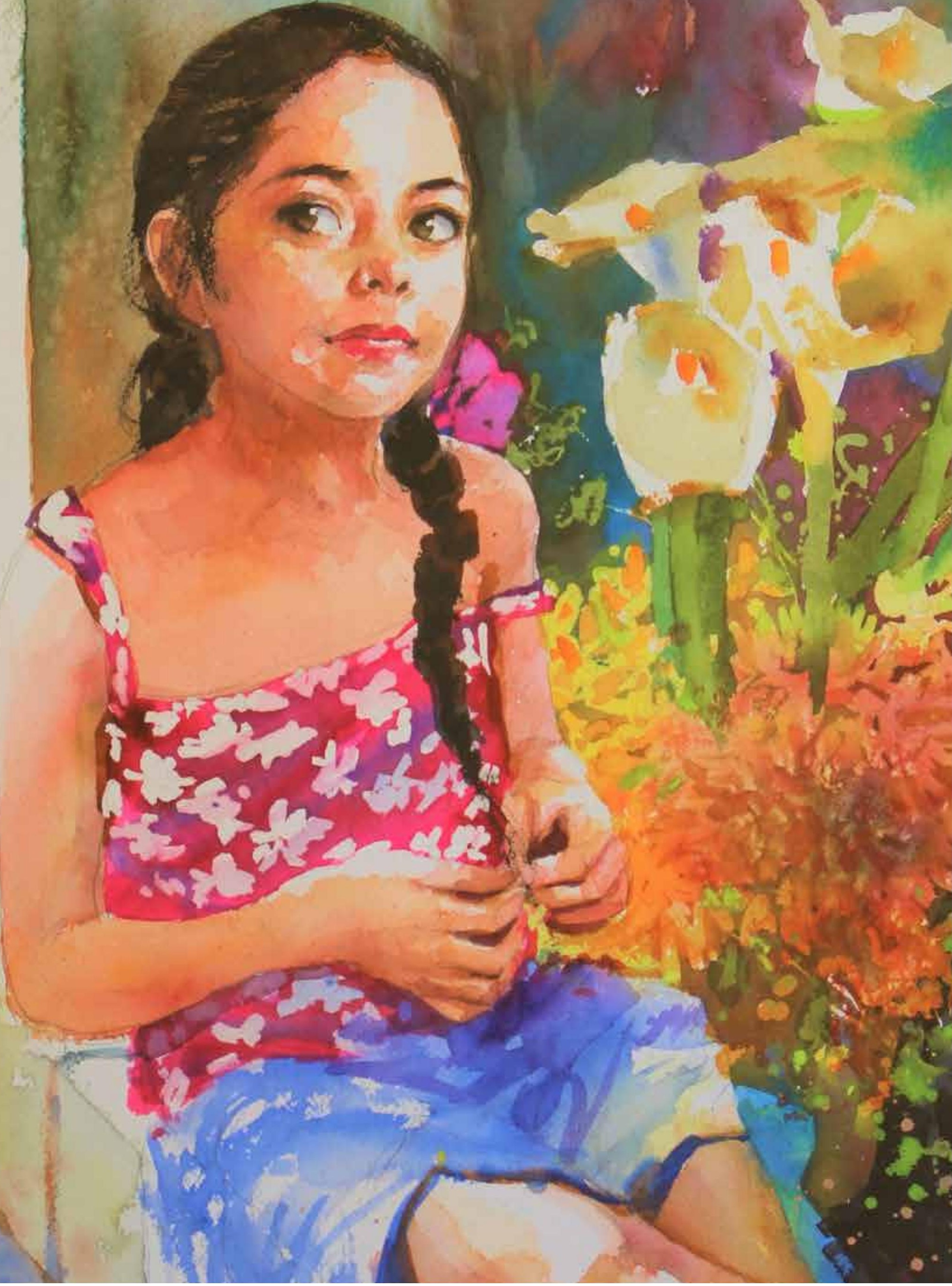




\section{Catástrofes naturales}

Con temblor la tierra aclama

al hombre que tenga piedad

con fuertes gritos exclama

que lo que haga no es nimiedad.

Son pedidos de auxilio

de un mundo descuidado

¿Tendremos que buscar exilio

por lo que bemos abandonado?

Las aguas también protestan

con lluvias desbordan los ríos.

Los bombres solo contestan

cuidando sus grandes señoríos.

Hacen cumbres y reuniones,

grandes cenas, fotos miles,

firmando falsas uniones

pero se protegen con misiles.

En Marte se busca vida

a Saturno cohetes lanza.

De nuestro planeta se olvida

dejándonos sin esperanza.

Es doble mensaje que dan:

por un lado, la advertencia,

lo que nos recomiendan

y en el otro la inclemencia.

Tsunamis, terremotos, inundación.

La tierra con esto se defiende.

Efecto invernadero y contaminación,

es el bombre que no entiende.

El ser bumano teme abora

cuando lo único necesario era

cuidar la obra creadora

porque es urgente y no espera.

Marcela Barrientos

Ulises Rivera

Técnica:

Acuarela 


\title{
4. Índice de vulnerabilidad ante efectos del cambio climático: Choluteca, Honduras
}

\author{
Ana Carolina Paz Delgado ${ }^{1}$ \\ DOI: https://doi.org/10.5377/pdac.v15i0.8116 \\ Recibido: 19/02/2019 - Aceptado:22/05/2019
}

\begin{abstract}
Resumen: Por su situación geográfica y características socioeconómicas, Honduras es considerado uno de los países más vulnerables del mundo ante los impactos del cambio climático; ya que su ubicación favorece el paso de los fenómenos climáticos extremos como huracanes y tormentas tropicales que, año con año azotan al país, situación que debilita su frágil economía frenando el desarrollo sostenible. Uno de los departamentos altamente afectados por el cambio climático, es el departamento de Choluteca, ubicado en la zona sur del país. En ese contexto el presente artículo analiza la situación de vulnerabilidad de los municipios del departamento de Choluteca ante los efectos del cambio climático mediante la construcción de un índice de vulnerabilidad. Se parte desde una visión retrospectiva, estimando los municipios que han sido más vulnerables, comprende el grado de exposición al riesgo, que enfrenta la población a eventos climáticos, así como a la sensibilidad al ser afectado por un desastre natural y la capacidad de adaptación tanto del gobierno local como de sus habitantes, desde un aspecto sociodemográfico y ambiental.

Mediante el análisis de indicadores sociodemográficos a nivel municipal, se identificó la población de los municipios de Choluteca más vulnerables ante el riesgo de los efectos del cambio climático, considerando que se han visto afectados por desastres naturales, con repercusiones en diversos aspectos de la vida. Uno de los impactos más sensibles es la seguridad alimentaria de la población, a la par del descenso en el nivel de ingreso de las familias que, ante este tipo de situaciones, toma la decisión de migrar a nivel interno o internacional, lo que tiene consecuencias en el descenso de la fecundidad y la composición del hogar.
\end{abstract}

Palabras claves: cambio climático, índice de vulnerabilidad, población y medio ambiente.

\section{Index of vulnerability to the effects of climate change: Choluteca, Honduras}

Abstract: Due to its geographical location and socioeconomic characteristics, Honduras is considered one of the most vulnerable countries in the world to the impacts of climate change; since its location favors the passage of extreme climatic phenomena such as hurricanes and tropical storms that, year after year, strike the country, a situation that weakens its fragile economy by slowing down sustainable development. One of the departments highly affected by climate change is the department of Choluteca, located in the southern part of the country. In this context, this article analyzes the situation of vulnerability of the municipalities of the department of Choluteca in regards to the effects of climate change through the construction of a vulnerability index. It starts from a retrospective view, estimating the municipalities that have been most vulnerable, including the degree of exposure to risk, which confronts the population to climatic events, as well as sensitivity to being affected by a natural disaster and the ability to adapt of both the local government as well as its inhabitants, from a socio-demographic and environmental aspect.

Through the analysis of sociodemographic indicators at the municipal level, the population of the Choluteca municipalities was identified as being most vulnerable to the risk of the effects of climate change, considering that they have been affected by natural disasters, with repercussions on various aspects of life. One of the most sensitive impacts is on the food security of the population, along with the decrease in the level of income of families that, faced with this type of situation, make the decision to migrate internally or internationally, which has consequences in the decline in fertility and the composition of households.

Keywords: climate change, vulnerability index, population, and environment.

\section{Introducción}

El cambio climático está asociado al aumento de emisiones de gases efecto invernadero (GEI) provenientes de actividades humanas, que alteran el funcionamiento natural del sistema climático del planeta Tierra, aumentando la temperatura, modificando los patrones de precipitación, incidiendo en la elevación del nivel del mar, reducción de glaciares y eventos climáticos extremos (SERNA y PNUD, 2013).
Dichos cambios representan una amenaza para la humanidad en general $y$, particularmente para los países más vulnerables, por los impactos en la producción agrícola y pesquera que pone en riesgo la seguridad alimentaria, los medios de vida en general, la salud, la infraestructura y el debilitamiento de la capacidad del ambiente para proveer recursos y servicios necesarios para el desarrollo.

1 Máster en Demografía y Desarrollo, Universidad Nacional Autónoma de Honduras. Ingeniera en Desarrollo Socioeconómico y Ambiente, Escuela Agrícola Panamericana "El Zamorano". Especialista en gestión estratégica, administración y ejecución de proyectos de desarrollo local. e-mail: ana.paz@unah.edu.hn 
Honduras, es considerado uno de los países más vulnerables del mundo a los impactos del cambio climático. El artículo se propuso como objetivo analizar la situación de vulnerabilidad de los municipios del departamento de Choluteca ante los efectos del cambio climático mediante la construcción de un índice de vulnerabilidad.

\section{Metodología}

La metodología que se utilizó para construir el índice de vulnerabilidad para el departamento de Choluteca es una adaptación del trabajo realizado por Heltberg y Bonch-Osmolovskiy (2011), acorde a la realidad departamental y a la disponibilidad de información (series históricas), variables o indicadores, entre otros aspectos. Esta adaptación describe cómo se traducen los conceptos de la exposición al riesgo, la sensibilidad, la capacidad de adaptación y la vulnerabilidad en los índices numéricos; variables usadas; y su agrupación en subíndices, y estos en un índice compuesto de vulnerabilidad que incorpora indicadores de ciencias sociales, demográficos y naturales (Anexo №1).

El índice de vulnerabilidad resulta del promedio simple de tres subíndices:

- Subíndice de exposición: se mide en función de la frecuencia en que la población se enfrenta a eventos de riesgo vinculados a los efectos del cambio climático, como deslizamientos, inundaciones, lluvias extremas, sequías y cambios radicales en las temperaturas máximas y mínimas. Este subíndice se compone de variables que miden la variabilidad climática, valores extremos de temperatura y precipitación, así como la ocurrencia de desastres naturales.

- Subíndice de sensibilidad: mide las condiciones que hacen que las personas sean vulnerables a los fenómenos extremos, afectando diferentes aspectos de su vida, como los referentes a cobertura agropecuaria, población dedicada a actividades agropecuarias, aspectos demográficos, economía y salud. Está compuesto por variables que miden condiciones agrícolas, relación de dependencia económica, salud, acceso a agua y sensibilidad relacionada con pérdidas económicas generadas por fenómenos hidrometeorológicos.
- Subíndice de la capacidad de adaptación: evalúa características educativas, de ingreso y la facultad institucional del gobierno nacional y local. Se compone de variables que miden educación, diversificación de los ingresos y desarrollo institucional a nivel local.

Se utilizaron promedios no ponderados simples de las variables normalizadas para los subíndices y promedios simples de subíndices para el índice general de vulnerabilidad ${ }^{2}$, incluyendo variables que representan cada uno los distintos aspectos de la vulnerabilidad para evitar tener los pesos implícitamente desiguales que resultarían si se incluyeron dos o más variables similares. Estas fueron definidas de acuerdo con la disponibilidad de información, para que el valor máximo de los subíndices de exposición y sensibilidad corresponda a la mayor vulnerabilidad, mientras que, para la capacidad de adaptación, el valor máximo corresponde a la vulnerabilidad más baja.

La construcción del índice comprendió la ejecución de tres etapas:

1. Se normalizaron todas las variables mediante una transformación lineal para ajustar los datos en el intervalo de 0-1, de esta forma permitir la comparación de los valores normalizados con conjuntos de datos y eliminar los efectos de influencias.

2. Posteriormente, se calculó cada subíndice mediante las siguientes fórmulas:

\section{Exposición:}

$E=((s d T 1+\ldots+s d T 12) / 12+(S D P 1+\ldots s d P 12) / 12+(r T 1+\ldots$ RT12) $/ 12+($ Nhot + Ncold $) / 2+($ Ndry + Ndisaster $) / 5$

\section{Dónde:}

SDTI = desviación estándar de la temperatura media mensual.

SDPI = desviación estándar de la precipitación total mensual.

$\mathrm{RTI}=$ promedio de los rangos entre las temperaturas promedio mensuales máxima y mínima.

Nhot $=$ frecuencia de meses extremadamente calurosos (porcentaje de registros por arriba de las temperaturas promedio mensuales máximas).

2 La metodología de los autores recomienda: "Los promedios simples asumen que todas las variables tienen igual peso. Promedios ponderados se pueden utilizar para partir de la asunción de pesos iguales, pero introducir la necesidad de "juicio de expertos" para determinar los pesos, introduciendo así un elemento más de la elección arbitraria. Pesos basados en regresión sólo son factibles cuando existe una medida objetiva de los resultados (en este caso la vulnerabilidad); este no es el caso aquí, ya que entonces no habría la necesidad de calcular el índice. Elegimos utilizar promedios no ponderados simples como el método más simple y menos arbitraria disponible". 
Ncold = frecuencia de meses extremadamente fríos (porcentaje de registros por abajo de las temperaturas promedio mensuales mínimas).

Ndry = frecuencia de ambientes extremos secos en el mismo mes del año (entre diciembre - abril, cero precipitaciones, entre mayo - noviembre aquellos registros donde ocurrió menos de dos desviaciones estándar de los milímetros, precipitados en el mismo mes durante los años de estudio).

Ndisaster $=$ frecuencia de desastres ocurridos entre 1988-2013.

\section{Sensibilidad:}

$S=(S 1+S 2) / 2+(S 3+S 4) / 2+(S 5+S 6) / 2+S 7 / 4$

\section{Dónde:}

S1 = porcentaje de cobertura agropecuaria.

S2 = porcentaje de agricultores, ganaderos y trabajadores agropecuarios.

S3 = ratio entre población menor a 15 años partido por el total de la población activa (15 a 64 años), por cien.

S4 = ratio entre población mayor o igual a 65 años divido por el total de la población activa (15 a 64 años), por cien.

S5 = índice de salud.

S6 = porcentaje de población sin acceso a fuentes de agua mejorada.

S7 = pérdidas monetarias por desastres ocurridos.

\section{Capacidad de adaptación:}

$A=(A 1+A 2)+(A 3+A 4+A 5 / 3) / 3$

\section{Dónde:}

A1 = índice de ingreso.

A2 = índice de educación.

A3 = grado de confianza en las personas (se puede confiar en la mayoría de las personas).

A4 = grado de confianza en el gobierno (ninguna).

A5 = corrupción y uso de coimas en el gobierno nacional (la mayoría de los funcionarios son corruptos).

Finalmente se obtuvo el índice de vulnerabilidad calculando la fórmula que comprende el resultado de los tres subíndices calculados previamente.

Vulnerabilidad $=1 / 3 *(($ Exposición + Sensibilidad + (1 - Capacidad de Adaptabilidad))
Un índice de valor alto indica mayor vulnerabilidad del municipio ante los efectos del cambio climático e índices de valor bajo indican lo contrario.

\section{- Limitantes para la construcción del índice de vulnerabilidad}

En la construcción del índice para explicar la relación causa y efecto de la vulnerabilidad de la población en estudio se identificaron las siguientes limitantes:

- La disponibilidad de información proporcionada por el Sistema Meteorológico Nacional de Honduras, fue la estación meteorológica de la cabecera departamental de Choluteca, que contiene el registro de la información sobre grados de temperatura y niveles de precipitación entre los años 1995 y 2014.

Debido a que no existen estaciones meteorológicas a nivel municipal, se utilizaron los registros disponibles para estas variables a nivel departamental, por lo tanto, se asignó el valor de la cabecera del departamento a todos los municipios que componen Choluteca (por ejemplo, el rango de temperatura promedio del municipio de Choluteca, se asignó a todos los municipios que componen el departamento).

- La aplicación de la metodología seleccionada tiene limitantes, una de ellas ocurre cuando la distribución de los datos no está bien comportada (no es una normal), el valor máximo o mínimo de la distribución puede ser muy extremo, esto hace que los demás valores asuman posiciones normalizadas en el extremo inferior o superior de la distribución, implicando la mayor ponderación de algunas variables sobre otras, por lo que algunos subíndices terminan aportando más a la vulnerabilidad que otros.

No obstante, dentro del análisis de variables, la forma en que los datos se distribuyen no precisa error en la estimación, debido a que contienen valores que representan la realidad y compilados por diferentes entes gubernamentales y organismos internacionales. Sin embargo, para evitar los valores atípicos en las series de datos observados, que no estuvieran distantes del resto de los datos y que no se vean afectadas las tablas restándole participación a otras variables claves en la determinación de la vulnerabilidad, se utilizó el valor promedio del valor real que permitió mejorar la estimación de las variables seleccionadas.

\section{Discusión de resultados}

La vulnerabilidad general de la población en el departamento de Choluteca está distribuida territorialmente a 
nivel municipal, bajo una escala de colores que indican los grados de vulnerabilidad: baja, moderada, media, alta y extrema ${ }^{3}$; que se aprecian en cada mapa. Esta medición resulta de una serie de factores que determinan la alta exposición de la población al riesgo, las condiciones sociales y demográficas en las que habitan, la baja capacidad económica, la limitada organización entre el gobierno local y población para impulsar planes de desarrollo municipal; factores explicativos de la vulnerabilidad en el departamento de Choluteca.

Los niveles de vulnerabilidad superiores están asociados a la exposición al riesgo que enfrentan los municipios cuando ocurren eventos relacionados al cambio climático. Esta situación se eleva cuando presentan un alto grado de sensibilidad donde se ven afectados por la variabilidad climática y, tienen niveles de adaptabilidad insuficientes, generando la vulnerabilidad de la población.

La mayoría de los municipios del departamento de Choluteca se encuentran en situación de vulnerabilidad media, alta y extrema, entre estos municipios están: El Triunfo, Marcovia, San Isidro, San Antonio de Flores, Duyure, Namasigue, El Corpus, Pespire, Orocuina, Concepción de María y Santa Ana de Yusguare, muestran una situación de vulnerabilidad extrema. Apacilagua una vulnerabilidad alta y, San José, una vulnerabilidad media. Según datos censales del 2013 estos municipios concentraban el 58\% de la población (Mapa №1).

Los municipios en situación de vulnerabilidad baja y moderada son: la cabecera departamental de Choluteca, San Marcos de Colón y Morolica, los cuales concentraban $42 \%$ de la población en el 2013. El municipio de Choluteca presenta una baja vulnerabilidad debido a que ha sido beneficiado con financiamiento de cooperantes internacionales y del gobierno de la República para desarrollar diferentes proyectos de mitigación, gestión de riesgos y desastres. El Gráfico №1 muestra los aportes no ponderados de los subíndices para cada municipio y el grado de magnitud de cada variable. La exposición al riesgo en los municipios del departamento de Choluteca se presenta con la frecuente ocurrencia de desastres, con mayor magnitud por inundaciones y deslizamientos, ocasionados por tormentas tropicales, lluvias extremas y el desbordamiento del río Choluteca.
A nivel municipal, la alta sensibilidad revela condiciones demográficas que los hacen particularmente vulnerables. Entre esas condiciones se puede mencionar la dependencia de la población menor de 15 años y la población mayor de 65 años sobre la población económicamente activa; asimismo, el deficiente índice de salud, el alto porcentaje de población sin acceso a fuentes de agua mejorada, la baja cobertura agropecuaria en la zona, asociada a la menor participación de la población dedicada a las actividades agropecuarias; esto aumenta la probabilidad de sufrir algún tipo de carencia.

La baja capacidad de adaptación presenta problemas debido al menor índice de ingreso y educación de la población, de igual manera, el bajo grado de confianza que las personas tienen respecto a los gobiernos locales, estos factores determinan o afectan el grado de vulnerabilidad.

Mapa №1 Departamento de Choluteca: índice de vulnerabilidad por municipios

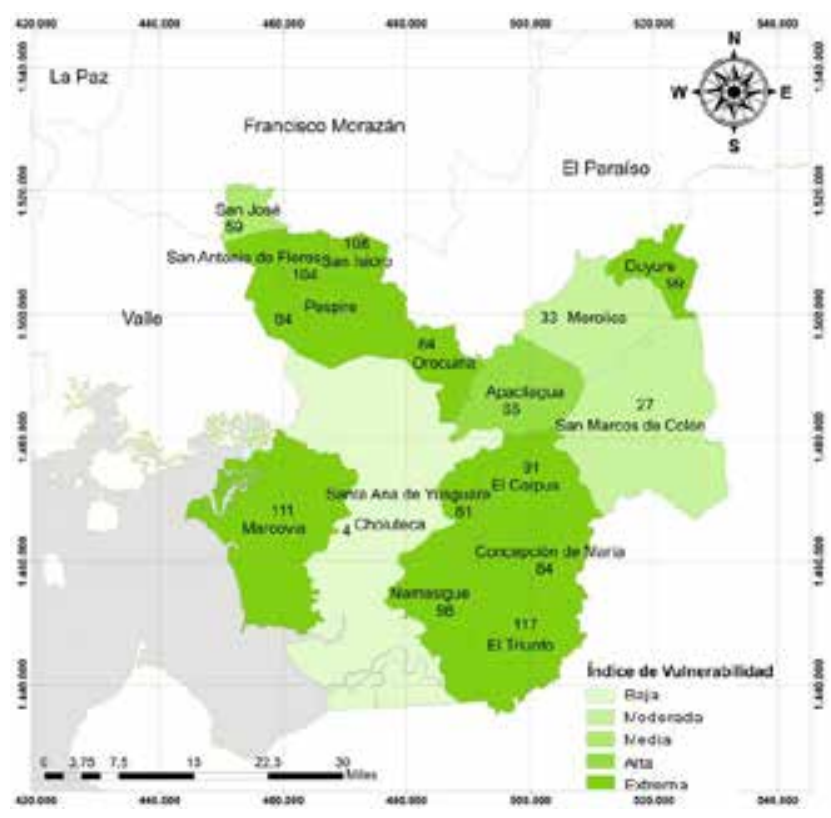

Fuente: Elaboración propia con base en el cálculo de la fórmula que comprende el resultado de los tres subíndices (exposición al riesgo, sensibilidad y capacidad de adaptación); con datos obtenidos del Servicio Meteorológico Nacional de Honduras (SMN), 1995-2014; Desinventar. Sistema de inventario de efectos de desastres, 1988-2013; Atlas Municipal Forestal y Cobertura de la Tierra, ICF, 2006 y 2014; XVII Censo de Población y VI Vivienda, 2013, Índice de

Desarrollo Humano, 2011; y Latinobarómetro, 2013.

3 Cada nivel en las escalas tiene una amplitud definida por la fórmula: Amplitud = (índice máximo - índice mínimo) /5, el límite superior de la escala "baja" se forma sumando el índice mínimo más una vez la amplitud; el límite de la escala "moderada" se realiza sumando al índice mínimo dos veces la amplitud y así sucesivamente. 
Gráfico Nº 1 Departamento de Choluteca: aportes no ponderados de los subíndices a la vulnerabilidad de la población por municipios.

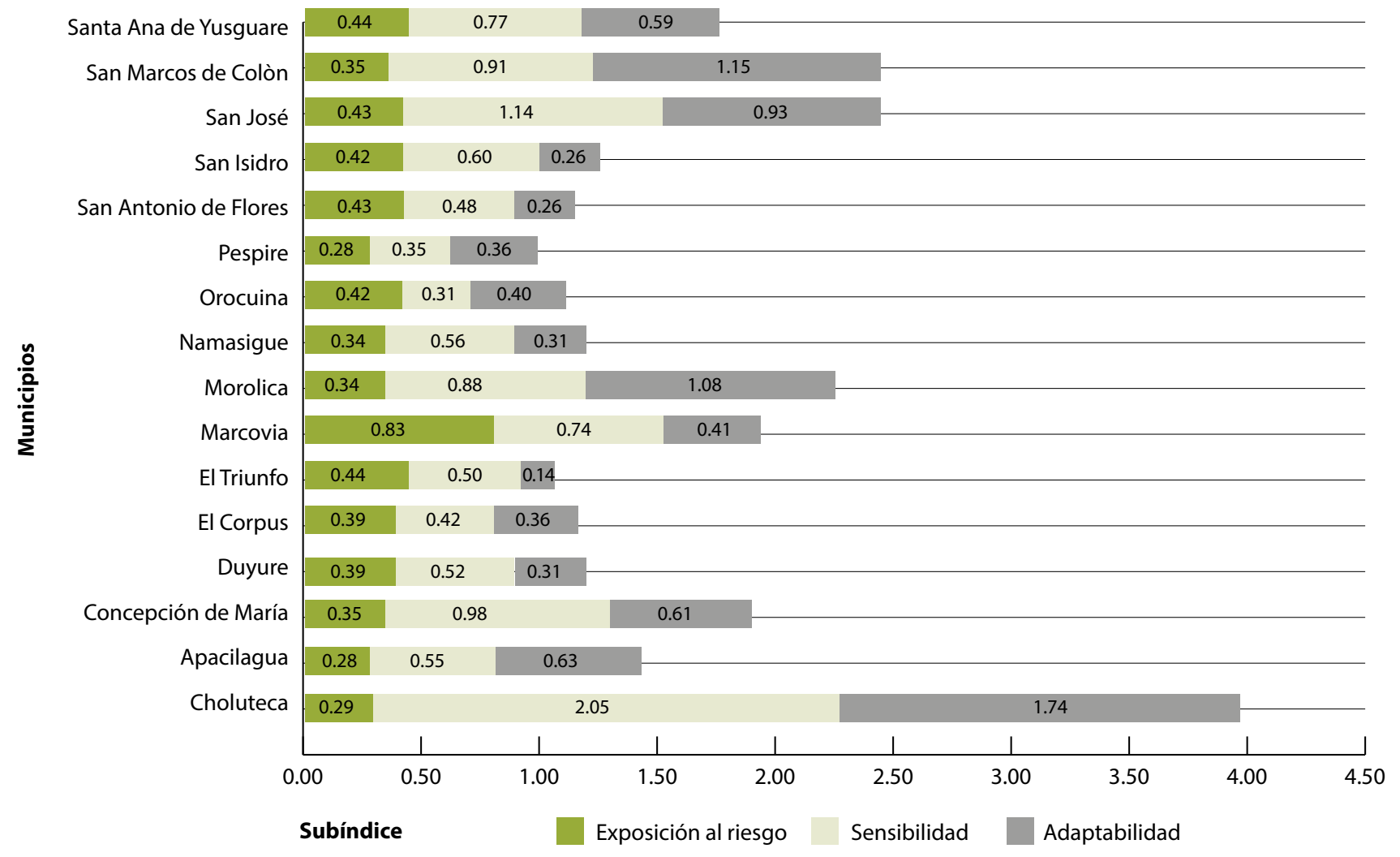

Fuente: Elaboración propia con base en el cálculo de la fórmula de cada subíndice; con datos obtenidos del Servicio Meteorológico Nacional de Honduras (SMN), 1995-2014; Desinventar. Sistema de inventario de efectos de desastres, 19882013; Atlas Municipal Forestal y Cobertura de la Tierra, ICF, 2006 y 2014; XVII Censo de Población y VI Vivienda, 2013, Índice de Desarrollo Humano, 2011; y Latinobarómetro, 2013.

\subsection{Exposición al riesgo por municipios del departa- mento de Choluteca}

La exposición mide el grado en que la población se enfrenta a eventos de riesgo vinculados a los efectos del cambio climático, como deslizamientos, inundaciones, lluvias extremas, sequías y cambios radicales en las temperaturas máximas y mínimas.

La mayoría de los municipios del departamento de Choluteca presentan una magnitud de exposición al riesgo moderada ya que han sido afectados por desastres ocurridos entre 1988-2013, tales como huracanes, tormentas tropicales, inundaciones, deslizamientos, marejadas y sequías. La frecuencia de los desastres contribuye a incrementar la vulnerabilidad local.

Estos municipios se encuentran expuestos a temperaturas altas extremas (durante el periodo de estudio analizado se han registrado temperaturas promedio de hasta $45^{\circ} \mathrm{C}$ ).

La presencia de ambientes extremos calurosos, fríos y secos en ciertas temporadas, generan consecuencias socioeconómicas en aquellas poblaciones con deficiencias notorias. En particular, las relacionadas a la baja disponibilidad de cobertura agropecuaria ${ }^{4}$ y una mayor población dedicada a actividades agropecuarias con grandes extensiones agrícolas, situación que pone en riesgo la seguridad alimentaria de la población, afectando dos de los componentes básicos: la disponibilidad de alimentos y acceso a alimentos, debido a que están ubicados en zonas vulnerables a cambios climáticos. Por ejemplo, durante un evento de inundación la población pierde hasta la totalidad de su producción. Unido a ello con los deslizamientos que cubren las vías de comunicación, se impide el acceso a mercados.

4 Es la relación entre la sumatoria de la superficie de tierras con cultivos anuales o transitorios, tierras en barbecho y/o descanso respecto la superficie total; para un año dado. 
El municipio de Marcovia presenta una exposición al riesgo extrema, debido a su ubicación geográfica, sus costas han sido afectadas por la frecuencia de desastres. Los municipios de El Triunfo, Santa Ana de Yusguare, San José, San Antonio de Flores, San Isidro y Orocuina, presentan una exposición al riesgo media. En cambio, Duyure, El Corpus, San Marcos de Colón, Concepción de María, Namasigue, Morolica, Choluteca, Apacilagua, y Pespire, presentan una magnitud moderada a la exposición al riesgo. La frecuencia de desastres ha sido significativa y la mayoría de estos municipios poseen una alta sensibilidad de ser afectados por un evento climático (Mapa №2).

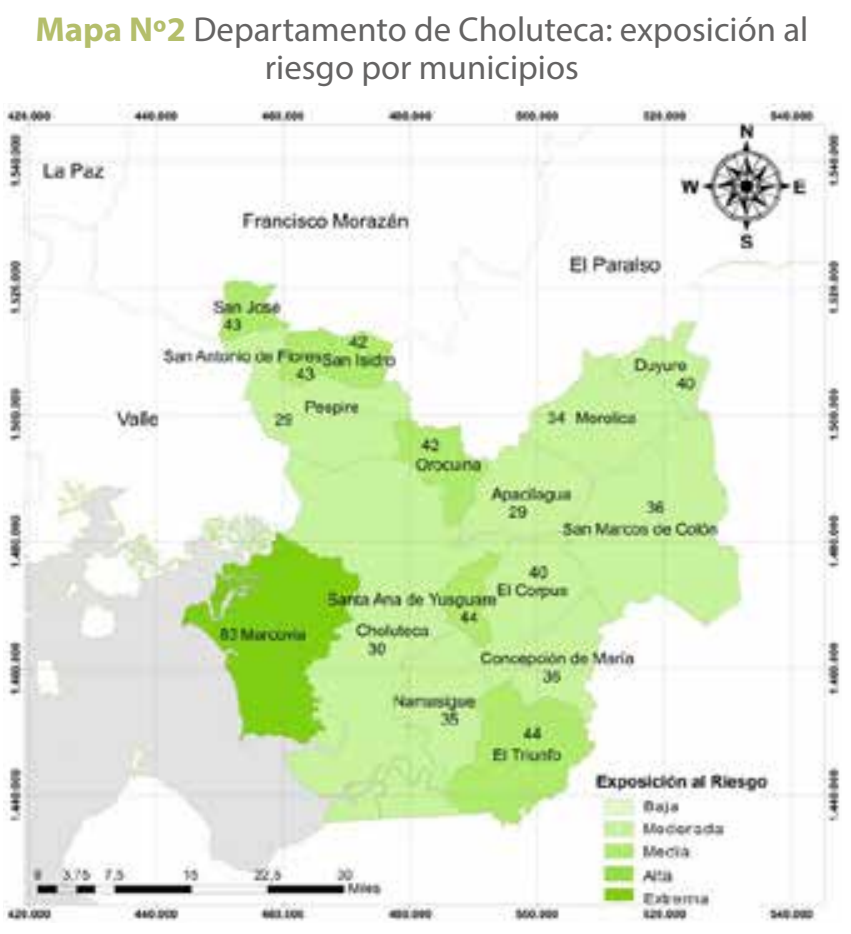

Fuente: Elaboración propia con base en datos del Servicio Meteorológico Nacional de Honduras (SMN), 1995-2014; y Desinventar. Sistema de inventario de efectos de desastres, 1988-2013.

\subsection{Sensibilidad por municipios del departamento de Choluteca}

La sensibilidad mide las consecuencias que tiene la exposición a los eventos vinculados al cambio climático en diferentes aspectos de la vida de la población, como los referentes a la cobertura agropecuaria, población dedicada a actividades agropecuarias, relación de dependencia económica y aspectos de salud.

Los últimos censos reflejan un comportamiento descendente de la tasa de dependencia demográfica en el departamento de Choluteca, sin embargo, sigue siendo alta; para el caso en 2013 fue de 69 personas lo que significa que por cada 100 personas en edad productiva hay 69 personas en edades inactivas (niños y adultos), es importante resaltar que se trata de una tasa de dependencia potencial pues no todas las personas menores de 15 años o mayores de 65 años están fuera del mercado laboral, ni todas las de 15-64 son activos.

Por otro lado, la población menor de 15 años representó el 35\%, de la población total del departamento de Choluteca, lo que es congruente con el proceso del bono demográfico donde las poblaciones dependientes disminuyen y la población económicamente activa aumenta. Sin embargo, este grupo de población hace particularmente sensible a la población en edad de trabajar en la medida que sus ingresos se ven afectados por la ocurrencia de eventos extremos, en este contexto, las posibilidades de recuperación dependerán de su fortaleza laboral, misma que es presionada por la dependencia económica de la población joven.

Contrario a lo que se observa en la población menor de 15 años, la población de 65 años va en aumento, en 2013 fue de $6 \%$. Honduras sigue siendo un país joven, sin embargo, el proceso de envejecimiento en Choluteca es moderado y la proporción de personas mayores de 65 años va en aumento, lo que hace pensar que es necesario identificar las prioridades y necesidades de las personas adultas para generar desarrollo y bienestar, estimulando políticas y acciones que beneficien a los grupos más vulnerables del país indistintamente del departamento en donde residan.

Los municipios con sensibilidad extrema, alta y media se ven afectados por las secuelas del cambio climático. Entre ellos están: Choluteca, San José, Concepción de María, San Marcos de Colón, Morolica, Santa Ana de Yesguare, Marcovia, San Isidro, Namasigue, Apacilagua, Duyure, El Triunfo, San Antonio de Flores y El Corpus, el territorio de estos municipios presenta una baja disponibilidad de cobertura agrícola y un número significativo de productores agropecuarios de pequeña escala, lo que da como resultado alta sensibilidad. Asimismo, el $28 \%$ de población sin acceso a fuentes de agua mejorada refleja deficiencias en el índice de salud que afectan su sensibilidad ante la ocurrencia de eventos climáticos.

En el caso de los municipios de Pespire y Orocuina, presentan una sensibilidad moderada. De igual forma poseen una baja cobertura agropecuaria y la población dedicada a las actividades agropecuarias es menor (Mapa №3). 


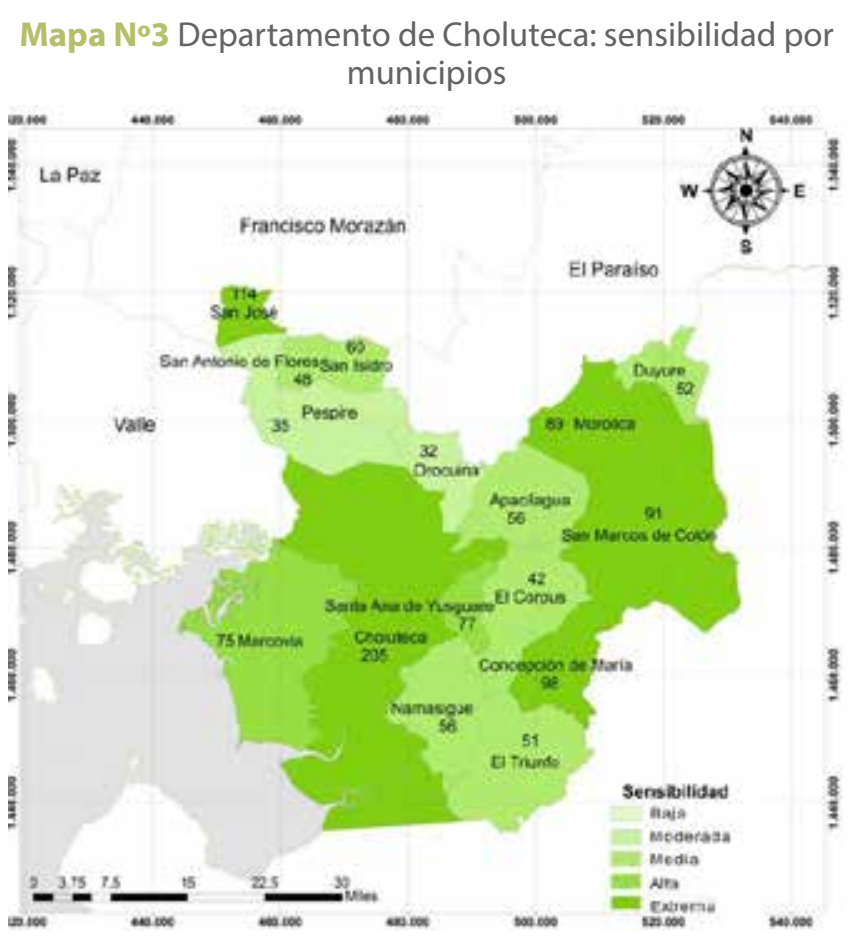

Fuente: Elaboración propia con base en datos del Atlas Municipal Forestal y Cobertura de la Tierra, ICF, 2006 y 2014; XVII Censo de Población y VI Vivienda, 2013, Índice de Desarrollo Humano, 2011 y Desinventar. Sistema de inventario de efectos de desastres, 1988-2013.

\subsection{Capacidad de adaptación por municipios del de- partamento de Choluteca}

La capacidad de adaptación es el potencial de una comunidad para ajustarse a los efectos del cambio climático a fin de moderar los daños potenciales, aprovechar las consecuencias positivas o soportar las consecuencias negativas. Para determinar esa capacidad se evalúan características educativas, de ingreso y la facultad de las instituciones del gobierno, nacional y local, para enfrentar la exposición y sensibilidad al riesgo.

Los municipios que presentan una extrema, alta y media capacidad de adaptación son: Choluteca, San Marcos de Colón, Morolica, San José; Apacilagua, Concepción de María, Santa Ana de Yusguare, Marcovia y Orocuina, al mostrar altos índices de ingreso y educación. Los gobiernos locales de estos municipios presentan mayor capacidad para implementar proyectos de adaptabilidad y mitigación.

En el caso de los municipios de Pespire, El Corpus, Duyure, Namasigue, San Isidro, San Antonio de Flores y El Triunfo, presentan baja y moderada capacidad de adaptación. Los bajos ingresos a escala local son entre otros, factores que contribuyen a la baja formación académica de la población que ha reducido la capacidad de absorber nuevas tecnologías que les permita diseñar estrategias efectivas para reducir su vulnerabilidad. Estos factores dificultan la preparación adecuada de sus habitantes para atenuar los efectos negativos y asegurar la recuperación inmediata ante los efectos adversos derivados de la exposición al cambio climático. Otro factor clave, es el nivel de confianza hacia otras personas y el gobierno local de los municipios antes expuestos, limitando la toma de decisiones para emprender proyectos (Mapa №4).

\section{Mapa №4 Departamento de Choluteca: capacidad de adaptación por municipios}

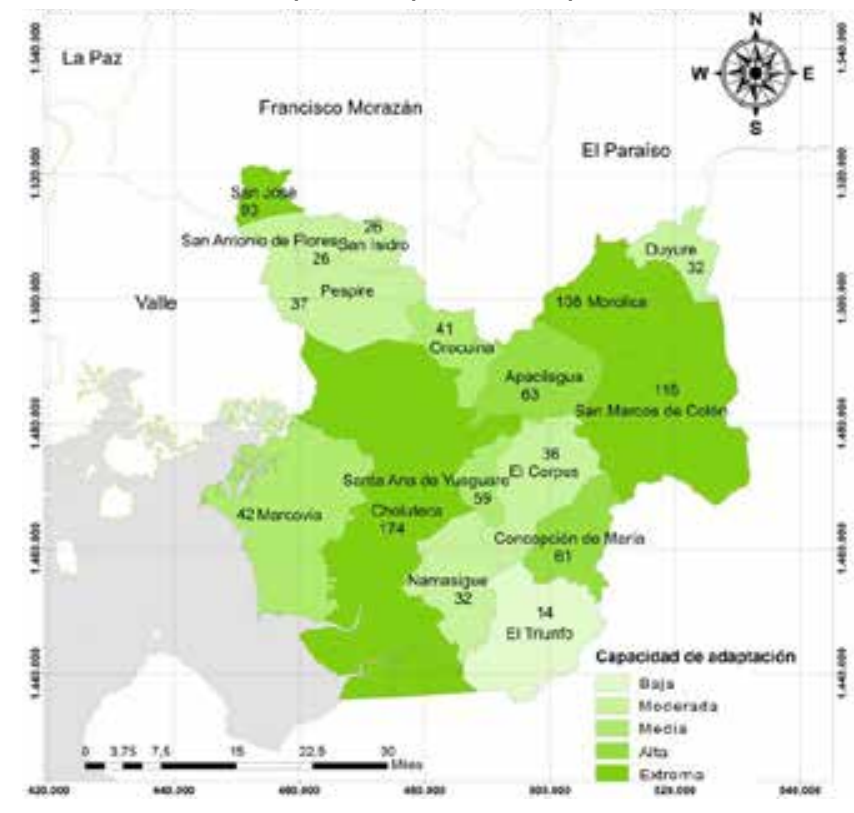

Fuente: Elaboración propia con base en datos al Índice de Desarrollo Humano, 2011 y Latinobarómetro, 2013.

\section{Conclusiones}

La población del departamento de Choluteca, en el período de 1988-2013, a nivel municipal ha sido afectada significativamente por fenómenos hidrometeorológicos, entre los cuales resaltan las inundaciones que han ocasionado pérdidas humanas y monetarias a nivel departamental. Las características sociodemográficas y los factores climáticos de los municipios analizados influyen en el grado de magnitud para que aumente o reduzca el impacto de un desastre natural.

El índice de vulnerabilidad estimado permitió identificar los municipios más vulnerables y los factores que condicionan esa vulnerabilidad. El enfoque de vulnerabilidad ante el cambio climático fortaleció la construcción de indicadores de vulnerabilidad combinando la evaluación cualitativa de la población, con una evaluación cuantitativa, basada en variables que miden numéricamente la ex- 
posición al riesgo, la sensibilidad de ser afectado por un evento climático $y$, en general, la falta de resiliencia social de la población.

Desde una visión retrospectiva la metodología aplicada identificó los municipios más vulnerables ante una amenaza específica, además de determinar qué factores contribuían a la vulnerabilidad. Esa información es útil para focalizar las acciones de prevención de riesgos y las medidas de mitigación y adaptabilidad, que permitan implementar políticas que mejoren la resiliencia de la población. Al mismo tiempo, informar a las autoridades centrales y locales sobre las características sociodemográficas y ambientales que inciden en la vulnerabilidad de la población ante efectos del cambio climático.

\section{Referencias bibliográficas}

- Desinventar. (2015). Sistema de inventario de efectos de desastres: Registros de Desastres y Pérdidas en Honduras. Disponible en: http://online.desinventar.org/?lang=spa

- Heltberg, R., y Bonch-Osmolovskiy, M. (2011). Mapping Vulnerability to Climate Change. Washington, DC, USA.

- ICF (2015). Atlas Municipal Forestal y Cobertura de la Tierra a nivel municipal del Departamento de Choluteca. Municipio del Distrito Central, Honduras.

- INE (2013). XVII Censo de Población y VI Vivienda a nivel municipal del Departamento de Choluteca. Tegucigalpa, Honduras.

- Latinobarómetro (2013). Análisis de Datos sobre La Democracia de Honduras. Providencia Santiago, Chile. Disponible en: http://www.latinobarometro.org/latOnline.jsp

- PNUD (2012). Informe sobre Desarrollo Humano Honduras 2011. Reducir la inequidad: un desafío impostergable. Tegucigalpa, Honduras.

- SERNA, y PNUD. (2013). Segunda Comunicación Nacional del Gobierno de Hondura ante la Convención Marco de las Naciones Unidas sobre Cambio Climático. Tegucigalpa, Honduras.

- SMN. (2015). Promedios Mensuales de Variables Climáticas del Departamento de Choluteca. Tegucigalpa, Honduras. 


\section{VIl. Anexos}

Anexo $N^{\circ} 1$. Variables para la construcción del Índice de Vulnerabilidad ante efectos del Cambio Climático

\begin{tabular}{|c|c|c|c|c|c|}
\hline Subíndice & ID & Variables incluidas en el índice & Año & $\begin{array}{l}\text { Información } \\
\text { utilizada }\end{array}$ & $\begin{array}{l}\text { Agregación } \\
\text { territorial }\end{array}$ \\
\hline \multirow{7}{*}{$\begin{array}{l}\text { Exposición al } \\
\text { riesgo }\end{array}$} & E1 & $\begin{array}{l}\text { Desviación estándar de la temperatura } \\
\text { media mensual }\end{array}$ & \multirow{6}{*}{$\begin{array}{l}1995- \\
2014\end{array}$} & \multirow{6}{*}{$\begin{array}{c}\text { Servicio } \\
\text { Meteorológi- } \\
\text { co Nacional } \\
\text { de Honduras } \\
\text { (SMN) }\end{array}$} & \multirow{6}{*}{$\begin{array}{l}\text { Municipio de } \\
\text { Choluteca }\end{array}$} \\
\hline & E2 & $\begin{array}{l}\text { Desviación estándar de la precipitación } \\
\text { total mensual }\end{array}$ & & & \\
\hline & E3 & $\begin{array}{l}\text { Promedio de los Rangos entre las tem- } \\
\text { peraturas promedio mensuales máx- } \\
\text { ima y mínima (Ran1 }+ \text { Ran2 }+\ldots .+ \\
\text { Ran12)/12 }\end{array}$ & & & \\
\hline & E4 & $\begin{array}{l}\text { Frecuencia de meses extremadamente } \\
\text { calurosos (porcentaje de registros por } \\
\text { arriba de las temperaturas promedio } \\
\text { mensuales máximas) }\end{array}$ & & & \\
\hline & E5 & $\begin{array}{l}\text { Frecuencia de meses extremadamente } \\
\text { fríos (porcentaje de registros por aba- } \\
\text { jo de las temperaturas promedio men- } \\
\text { suales mínimas) }\end{array}$ & & & \\
\hline & E6 & $\begin{array}{l}\text { Frecuencia de ambientes extremos } \\
\text { secos en el mismo mes del año (en- } \\
\text { tre Dic.-Abr., cero Precipitaciones, en- } \\
\text { tre May-Nov aquellos registros donde } \\
\text { ocurrió menos de dos desviaciones } \\
\text { estándar de los mm. Precipitados en el } \\
\text { mismo mes durante los años de estu- } \\
\text { dio) }\end{array}$ & & & \\
\hline & E7 & $\begin{array}{l}\text { Frecuencia de desastres naturales } \\
\text { ocurridos. }\end{array}$ & $\begin{array}{l}1988- \\
2013\end{array}$ & $\begin{array}{l}\text { Desinventar. } \\
\text { Sistema de } \\
\text { inventario de } \\
\text { efectos de } \\
\text { desastres }\end{array}$ & Por municipio \\
\hline
\end{tabular}




\begin{tabular}{|c|c|c|c|c|c|}
\hline Subíndice & ID & Variables incluidas en el índice & Año & $\begin{array}{l}\text { Información } \\
\text { utilizada }\end{array}$ & $\begin{array}{l}\text { Agregación } \\
\text { territorial }\end{array}$ \\
\hline \multirow{7}{*}{ Sensibilidad } & S1 & Porcentaje de cobertura agropecuaria & 2014 & $\begin{array}{l}\text { Atlas Munici- } \\
\text { pal Forestal y } \\
\text { Cobertura de la } \\
\text { Tierra, ICF }\end{array}$ & Por municipio \\
\hline & S2 & $\begin{array}{l}\text { Porcentaje de Agricultores, ganaderos } \\
\text { y trabajadores agropecuarios }\end{array}$ & \multirow{3}{*}{2013} & \multirow{3}{*}{$\begin{array}{l}\text { XVII Censo de } \\
\text { Población y VI } \\
\text { Vivienda, } 2013\end{array}$} & \multirow{3}{*}{ Por municipio } \\
\hline & S3 & $\begin{array}{l}\text { Ratio entre población menor a } 15 \text { años } \\
\text { dividido por el total de la población ac- } \\
\text { tiva ( } 15 \text { a } 64 \text { años). Por cien }\end{array}$ & & & \\
\hline & S4 & $\begin{array}{l}\text { Ratio entre Población mayor o igual a } \\
65 \text { años partido por el total de la po- } \\
\text { blación activa ( } 15 \text { a } 64 \text { años). Por cien }\end{array}$ & & & \\
\hline & S5 & Índice de Salud & 2011 & $\begin{array}{l}\text { Índice de } \\
\text { Desarrollo } \\
\text { Humano }\end{array}$ & Por municipio \\
\hline & S6 & $\begin{array}{l}\text { Porcentaje de Población sin acceso a } \\
\text { fuentes de agua mejorada }\end{array}$ & 2006 & $\begin{array}{l}\text { Atlas Munici- } \\
\text { pal Forestal y } \\
\text { Cobertura de la } \\
\text { Tierra, ICF }\end{array}$ & Por municipio \\
\hline & S7 & $\begin{array}{l}\text { Pérdidas económicas por desastres } \\
\text { ocurridos }\end{array}$ & $\begin{array}{l}1988- \\
2013\end{array}$ & $\begin{array}{l}\text { Desinventar } \\
\text { Sistema de } \\
\text { inventario de } \\
\text { efectos de } \\
\text { desastres }\end{array}$ & Por municipio \\
\hline \multirow{5}{*}{ Adaptabilidad } & A1 & Índice de Ingreso & & & \\
\hline & A2 & Índice de Educación & & Humano & \\
\hline & A3 & $\begin{array}{l}\text { Grado de confianza } \\
\text { en las personas } \\
\text { (Se puede confiar en la mayoría de las } \\
\text { personas) }\end{array}$ & \multirow{3}{*}{2013} & \multirow{3}{*}{$\begin{array}{l}\text { Latinobaróme- } \\
\quad \text { tro }\end{array}$} & \multirow{3}{*}{ A nivel de país } \\
\hline & A4 & $\begin{array}{l}\text { Grado de confianza en El Gobierno } \\
\text { (Ninguna) }\end{array}$ & & & \\
\hline & A5 & $\begin{array}{l}\text { Corrupción y uso de coimas en El Go- } \\
\text { bierno nacional (La mayoría de los fun- } \\
\text { cionarios son corruptos) }\end{array}$ & & & \\
\hline
\end{tabular}

